\title{
Potentially positive ageing-related variations of medial smooth muscle cells in the saphenous veins used as aortocoronary bypass grafts
}

\author{
Bartlomiej Perek$^{1}$, Agnieszka Malinska², Jerzy Gasowski ${ }^{3}$, Danuta Ostalska-Nowicka ${ }^{4}$, \\ Anna Perek ${ }^{5}$, Marek Jemielity', Maciej Zabel², Michal Nowicki²
}

${ }^{1}$ Department of Cardiac Surgery and Transplantology, Poznan University of Medical Sciences, Poland

${ }^{2}$ Department of Histology and Embryology, Poznan University of Medical Sciences, Poland

${ }^{3}$ Department of Internal Medicine and Gerontology, Jagiellonian University, Medical College, Krakow, Poland

${ }^{4}$ Department of Pediatric Cardiology and Nephrology, Poznan University of Medical Science, Poland

${ }^{5}$ Department of Anesthesiology and Intensive Therapy, Poznan University of Medical Sciences, Poland

\begin{abstract}
Introduction. Currently, elderly people constitute a large proportion of patients undergoing coronary artery bypass grafting (CABG). Activated smooth muscle cells in the tunica media of saphenous vein (SV) grafts are thought to play a key role in the formation of neointima and development of occluding atherosclerotic plaques. The aim of this study was to identify ageing-related variations in the expression of the smooth muscle cells proteins that may impact on patency rate of the grafts and the CABG outcomes.

Material and methods. The study involved 216 consecutive patients with the mean of $62.7 \pm 8.4$ years who underwent isolated CABG with at least one SV aortocoronary bypass graft. Expression of $\alpha$-smooth muscle actin ( $\alpha$-SM actin), smooth muscle-myosin heavy chain (SM-MHC), calponin (CALP), cytokeratin 8 (CK-8), metalloproteinase-2 (MMP-2) and tissue inhibitors of metalloproteinases-2 and -3 (TIMP-2, TIMP-3) in the SV wall was assessed by immunohistochemistry and correlated with the age of patients.

Results. Calponin and $\alpha$-SM actin were expressed in all studied SV transplants. SM-MHC immunoreactivity was observed in SV segments in $68.5 \%$ of patients, whereas MMP-2a and TIMPs expression was found in $75 \%$ of cases. In more than $50 \%$ of analyzed SV transplants, no expression of cytokeratin- 8 was found. Moderate correlations between preexisting expressions of either cytoskeletal or hemostatic proteins in the tunica media of the SV grafts and the age of CABG patients were demonstrated. They were positive for SM-MHC $(r=0.494)$, CALP $(r=0.548)$, TIMP-2 $(r=0.413)$ and TIMP-3 $(r=0.406)$ whereas negative for CK-8 $(r=-0.528)$ and MMP-2 ( $\mathrm{r}=-0.417)$.

Conclusions. Age-dependent decreases in the expression of MMP-2 and CK-8 accompanied by increases in expression of SM-MHC, TIMP-2 and TIMP-3 may promote SV graft patency and, thus, suggest a rationale for common use of SV grafts in the elderly. (Folia Histochem Cytobiol. 2016, Vol. 54, No. 2, 91-98).
\end{abstract}

Key words: venous aortocoronary grafts; smooth muscle cells; $\alpha$-SM actin; calponin; cytokeratin 8 ; SM-MHC; MMP-2; TIMP-2; TIMP-3 


\section{Introduction}

Currently, the elderly constitute a large proportion of patients undergoing invasive procedures, including coronary artery bypass grafting (CABG) [1]. Human ageing is characterized by time-dependent, deleterious changes in microstructure and function of the organs and tissues, including also cardiovascular system [2]. Much of morbidity and mortality in the elderly has been attributed to atherosclerosis-induced acute ischemic events that lead to myocardial infarction [3]. However, little is known about ageing-induced functional and microstructural changes in the saphenous vein (SV). Knowledge about ageing-related preexisting variations in the structure and function of the SV segments, particularly those without macroscopic abnormalities (considered as normal by surgeons), seems to be of a paramount importance for the fate of venous grafts after their placement into the arterial system.

Mid-term and late SV graft failure is determined by formation of the neointima [4]. Although, many local and systemic factors have an impact on its development, this process always involves smooth muscle cells (SMCs) located in the tunica media of the SV segment [5]. SMCs release matrix metalloproteinases (MMPs) that degrade extracellular matrix (ECM) [6]. They also produce tissue inhibitors of metalloproteinases (TIMPs), and a balance between MMPs and TIMPs eventually determines the local homeostasis of the ECM [7]. Moreover, the population of venous SMCs was reported to be heterogeneous regarding metabolic, proliferative and chemotactic activities [8]. Subtypes of SMCs were characterized by altered expression of smooth muscle proteins such as $\alpha$-smooth muscle actin ( $\alpha$-SM actin), smooth muscle myosin heavy chain (SM-MHC), calponin (CALP) and cytokeratin 8 (CK-8) [8]. These proteins were found to be associated with various stages of SMCs differentiation. SM-MHC and CALP are expressed predominantly in the mature SMCs whereas CK-8 expression is typical for fetal and poorly differentiated cells $[8,9]$.

Thus, the aim of the present study was to evaluate the expression of the selected cytoskeletal $(\alpha$-SMA, SM-MHC, CALP, CK-8) and homeostatic (MMP-2, TIMP-2, TIMP-3) proteins in the tunica media of the SV grafts in relation to the age of patients undergoing isolated non-emergency CABG that could have potential impact on long-term outcomes.

\section{Material and methods}

Study group. The Local Ethics Committee has approved the study protocol (No. 1201/08) and informed written consent
Table 1. Selected preoperative demographic and clinical data

\begin{tabular}{|l|c|}
\hline & Patients $N=\mathbf{2 1 8}$ \\
\hline Age (years) & $62.5 \pm 8.7$ \\
\hline Gender (male/female) & $168 / 50$ \\
\hline Mean BMI & $29.3 \pm 4.1$ \\
\hline Obesity (BMI > 30) & $92(42.2 \%)$ \\
\hline Stages of CAD & \\
\hline Stable angina & $180(82.6 \%)$ \\
Unstable angina & $38(17.4 \%)$ \\
History of infarct & $140(64.2 \%)$ \\
Previous PCI & $66(30.3 \%)$ \\
\hline Arterial hypertension & $162(74.3 \%)$ \\
\hline Diabetes mellitus & $98(45.4 \%)$ \\
\hline Hyperlipidemia & $92(42.4 \%)$ \\
\hline PVD & $50(22.9 \%)$ \\
\hline Neurological events & $18(8.3 \%)$ \\
\hline
\end{tabular}

Categorical variables are presented as numbers and percentages (\%) and continuous variables as means \pm standard deviation. Abbreviations: $\mathrm{BMI}$ — body mass index; $\mathrm{CAD}$ — coronary artery disease; $\mathrm{PCI}$ - percutaneous coronary intervention; PVD — peripheral vascular disease.

from each study participant was obtained. Two hundred sixteen consecutive patients ( 167 males, $77.3 \%$ and 49 females, $22.7 \%$ ) with a mean \pm SD age of $62.3 \pm 8.7$ year (range 42-85) who underwent primary isolated non-emergent CABG with at least one venous aortocoronary bypass graft were enrolled in this study. Patients with any pathologies of the peripheral veins were excluded from the study. Basic preoperative data are summarized in Table 1.

Intraoperative sample collection and preparation. The most distal surplus segments of the SV (at least $1.5-2.0 \mathrm{~cm}$ in length) were saved for the histological studies. In the operating room, the SV segments were rinsed with $0.9 \% \mathrm{NaCl}$ at the room temperature, slightly dilated and fixed in Bouin's solution for 2 or 3 hours. Then they were dehydrated, embedded in paraffin and cut into five micrometer-thick sections.

Immunohistochemistry. All immunohistochemical (IHC) analyses employed the Dako REAL ${ }^{\mathrm{TM}}$ EnVision $^{\mathrm{TM}}$ Detection System, Peroxidase/DAB, Rabbit/Mouse, K5007 (Dako, Glostrup, Denmark). After deparaffinization with xylene and gradual rehydration the endogenous peroxidase activity was blocked with $10 \%$ hydrogen peroxide (v/v). Then the sections were incubated with the following mouse anti-human monoclonal specific antibodies: anti- $\alpha$-SM actin (M0851, Dako), anti-SM-MHC (MAB13431, Dako), anti-CALP (M3556, Dako), anti-CK-8 (ab9023; Abcam, Cambridge, UK), anti-MMP-2 (MAB13431, Millipore, Darmstadt, Ger- 
many), anti-TIMP-2 (MAB3310, Millipore), anti-TIMP-3 (MAB3318, Millipore) for $18 \mathrm{~h}$ at $4^{\circ} \mathrm{C}$ and then for $60 \mathrm{~min}$ at room temperature. The samples were rinsed and then incubated for $60 \mathrm{~min}$ at room temperature with a dextran coupled with peroxidase molecules and goat secondary antibody molecules against mouse immunoglobulins (30 min at room temperature). The peroxidase reaction was developed using 0.5\% 3-3' diaminobenzidine (DAB; Sigma-Aldrich, St. Louis, MO, USA).

The intensity of cytoplasmic expression of the proteins was assessed using the semi-quantitative IRS (immune-reactive score) scale according to Remmele and Stenger [10]. It takes into account the percentage of positive cells (scale from 0 to 4 ) and the intensity of the color reaction (scale from 0 to 3 ) and final score that ranges from 0 to 12 is a product of scores given for individual traits. On the base of IRS, the expression of the cytoplasmic proteins was defined as negative (IRS 0-1), positive weak (IRS 2-3), moderate (IRS 4-6) or strong (IRS 8-12). All tissue sections were analyzed using an AxioImager Z.1 light microscope and representative images were captured with an attached AxioCam MRc5 digital camera (Carl Zeiss MicroImaging GmbH, Göttingen, Germany).

For uniformity, IHC analyses of the expression of proteins in each vessel section were done within 10 representative microscopic fields (magnification $\times 200$ ). The assessment of staining was evaluated blind and independently by two pathologists on the coded samples that included also both negative and positive controls. The negative controls consisted of the sections incubated with non-immune IgG1 (X0931, Dako) and the sections in which the primary or secondary antibody was omitted. In addition, the serial sections were stained in the subsequent experiments as positive controls to determine the consistency of staining.

Transmission electron microscopic study protocol. Material fixed in $2.5 \%$ glutaraldehyde in phosphate buffer $(0.05 \mathrm{M}$, $\mathrm{pH}$ 7.4) was post-fixed in $1 \% \mathrm{OsO}_{4}$ in the same buffer, dehydrated and embedded in Araldite (Polysciences Europe, Hirschberg an der Bergstrasse, Germany). Semithin and ultrathin sections were cut on a Leica Ultracut UCT (Leica Microsystems, Nussloch, Germany). The $0.3 \mu \mathrm{m}$-thick sections were stained with toluidine blue and examined under an Olympus BX50 light microscope (Olympus, Tokyo, Japan). Ultrathin sections were mounted on mesh nickel grids and then counterstained with uranyl acetate and lead citrate. These sections were examined using a JEM-1010 (JEOL, Tokyo, Japan) transmission electron microscope.

Statistical analysis. The Shapiro-Wilk W test for normality was performed for all continuous variables. When the values were normally distributed, they were expressed as the means \pm standard deviations. Categorical data were expressed as the number $(n)$ and percentages $(\%)$. The correlations be- tween results of analysis of immunohistochemically assessed expression of tissue proteins in the tunica media and the age of CABG patients were tested using Spearman's rank correlation and linear regression from which adjusted partial correlation coefficients were derived. The correlation coefficients were interpreted using the scale provided by Salkin, where an $\mathrm{r}$ between 0.8 and 1.0 (or -0.8 and -1.0 ) is defined as very strong, between 0.6 and 0.8 - as strong, between 0.4 and 0.6 - as moderate, between 0.2 and 0.4 - as weak and between 0.0 and 0.2 - as very weak or no relationship [11]. A P value $<0.05$ was considered statistically significant. Data management and statistical analyses were performed with Statistica 9.0 for Windows (StatSoft, Inc., Tulsa, OK, USA).

\section{Results}

\section{Expression of SMCs cytoskeletal proteins}

We found that $\alpha$-SM actin was expressed in all studied SV grafts. Its expression was strong or moderate within cytoplasm of the media's SMCs.

SM-MHC was not expressed in the SV segments obtained from 68 patients $(31.5 \%)$ whereas strong (IRS $\geq 8$ ) was found in only 10 cases $(4.6 \%$ ) (Figure 1$)$. In the other specimens, immunostaining of SM-MHC was estimated as weak or moderate. If present, SM-MHC was localized regularly within the cytoplasm of SMCs.

Calponin was expressed in all studied SV transplants. In the majority of the SV samples $(n=202,93.5 \%)$, its cytoplasmic presence within SMCs was regarded as moderate or strong. It was not seen within tunica adventitia or connective tissue present in the tunica media.

In more than $50 \%$ of analyzed SV transplants, no expression of cytokeratin-8 (IRS 0-1) (Figures 1 and 2) was observed. Otherwise, only in 24 cross-sections of the SV grafts $(11.1 \%)$ it was assessed as strong in the tunica media (Figure 2). Similarly to SM-MHC expression, CK-8 immunoreactivity was observed in the cytoplasm of SMCs.

We found moderate correlation between IHC expression of cytoskeletal proteins in the tunica media of the SV grafts and the age of CABG patients. These correlations were significantly positive for SM-MHC $(\mathrm{r}=0.494 ; \mathrm{P}<0.05)$ and CALP $(\mathrm{r}=0.548 ; \mathrm{P}<0.05)$, but negative for CK-8 tissue immunostaining $(\mathrm{r}=-0.528 ; \mathrm{P}<0.05)$. An exception was a very weak $(\mathrm{r}=-0.144 ; \mathrm{P}<0.05)$ correlation between the age of SV recipients and the expression of $\alpha$-SM actin in the tunica media of SV.

\section{Expression of matrix metalloproteinase-2 and tissue inhibitors of metalloproteinases}

In a quarter of the SV specimens, no IHC expression of MMP-2 and TIMPs was found (Figure 3). The majority of SV segments exhibited weak or moderate 


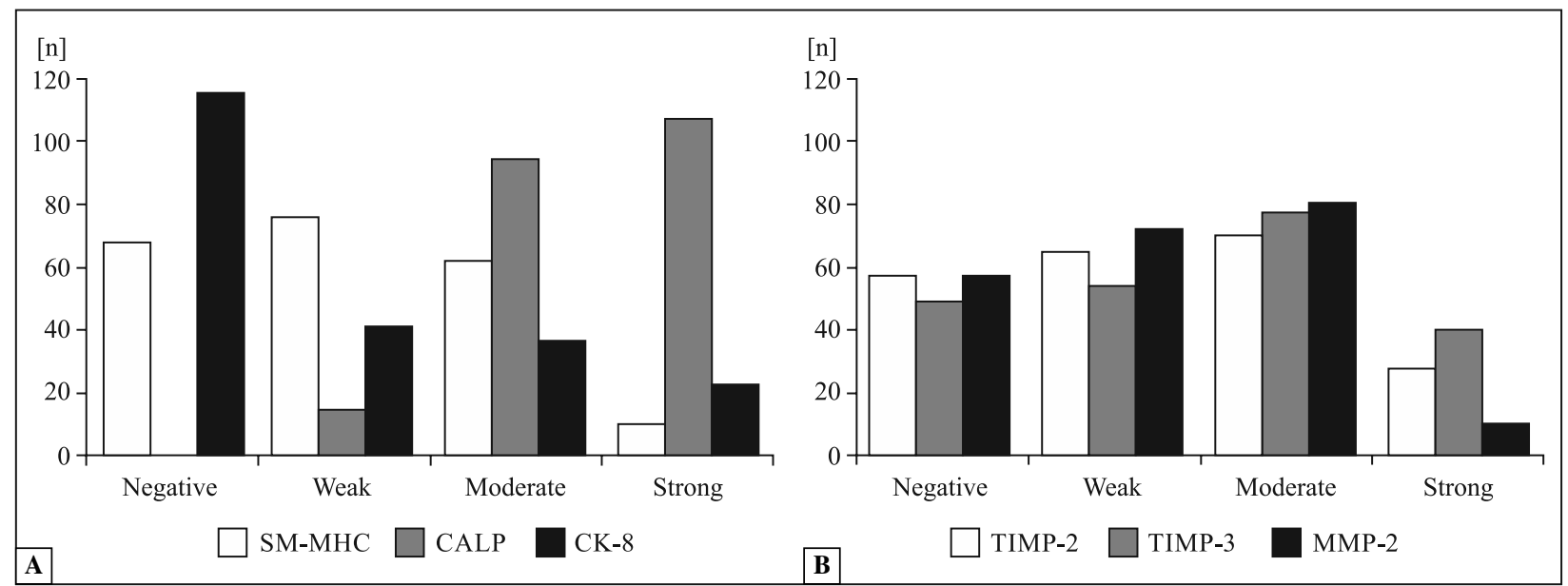

Figure 1. Semiquantitative assessment of the expression of cytoskeletal (A) and homeostatic (B) proteins in the tunica media of the saphenous vein grafts. Sections of the saphenous vein of 216 patients undergoing coronary artery bypass grafting $(\mathrm{CABG})$ were stained by immunochistochemical (IHC) method and the intensity and range of reactions were assessed as described in Material and methods. Abbreviations: CALP - calponin; CK-8 - cytokeratin 8; MMP — metalloproteinase; SM-MHC — smooth muscle myosin heavy chain; TIMP — tissue inhibitor of metalloproteinase.

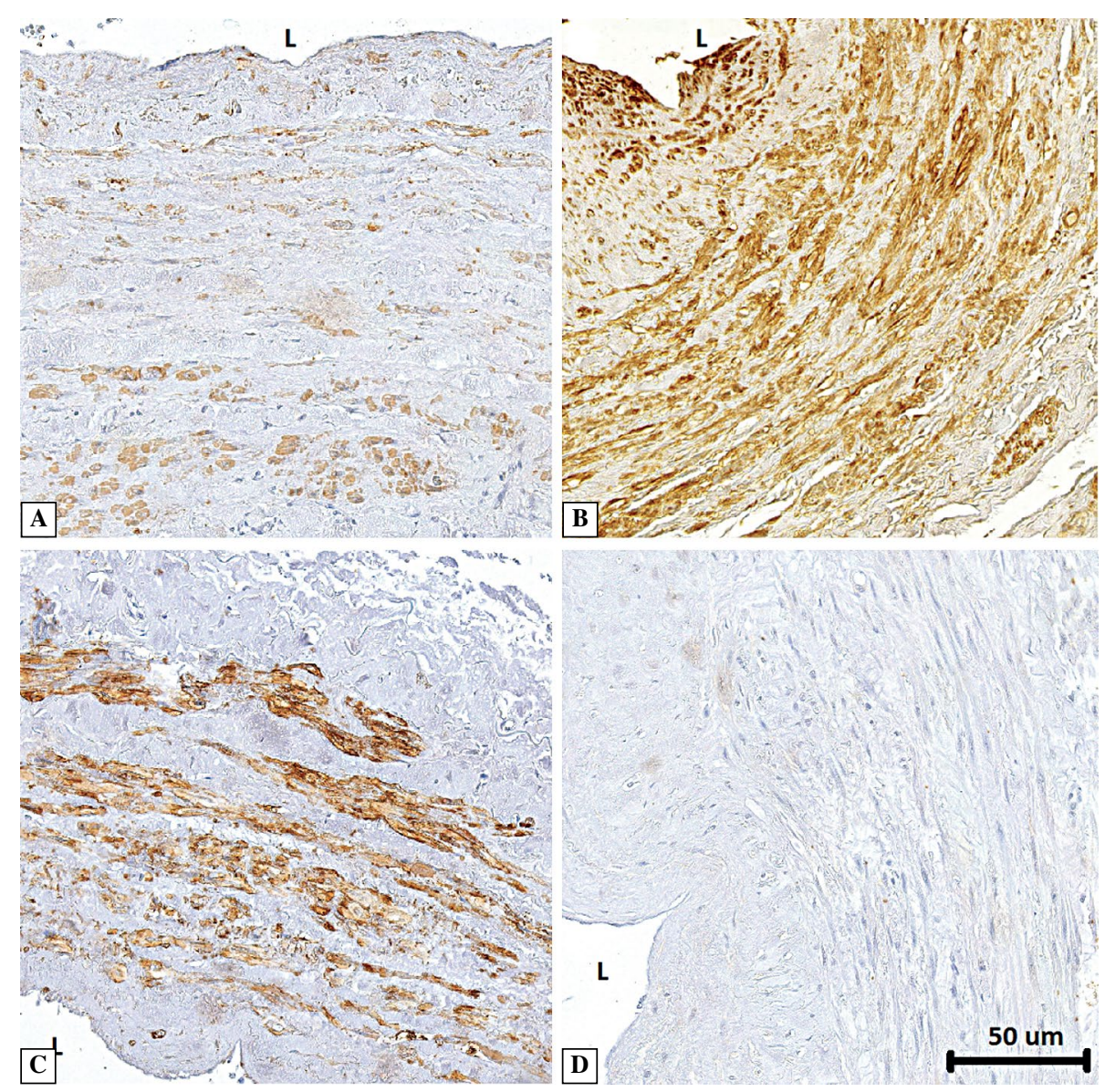

Figure 2. Immunoreactivity of the cytoskeletal proteins in the tunica media of the saphenous vein grafts of middle age and elderly patients. A, C. Section of saphenous vein (SV) segment of 47-year-old patient. Weak immunoreactivity of SM-MHC (IRS score 2) (A), and strong expression of CK-8 (IRS score 9) (C); B, D. Section of SV of 76-year-old patient. Strong expression of SM-MHC (IRS score 8) (B), and negative immunostaining for CK-8 (IRS score 0) (D). Abbreviations as for Figure $1 ; \mathrm{L}-$ lumen of the SV graft. Objective magnification: $\times 20$. 


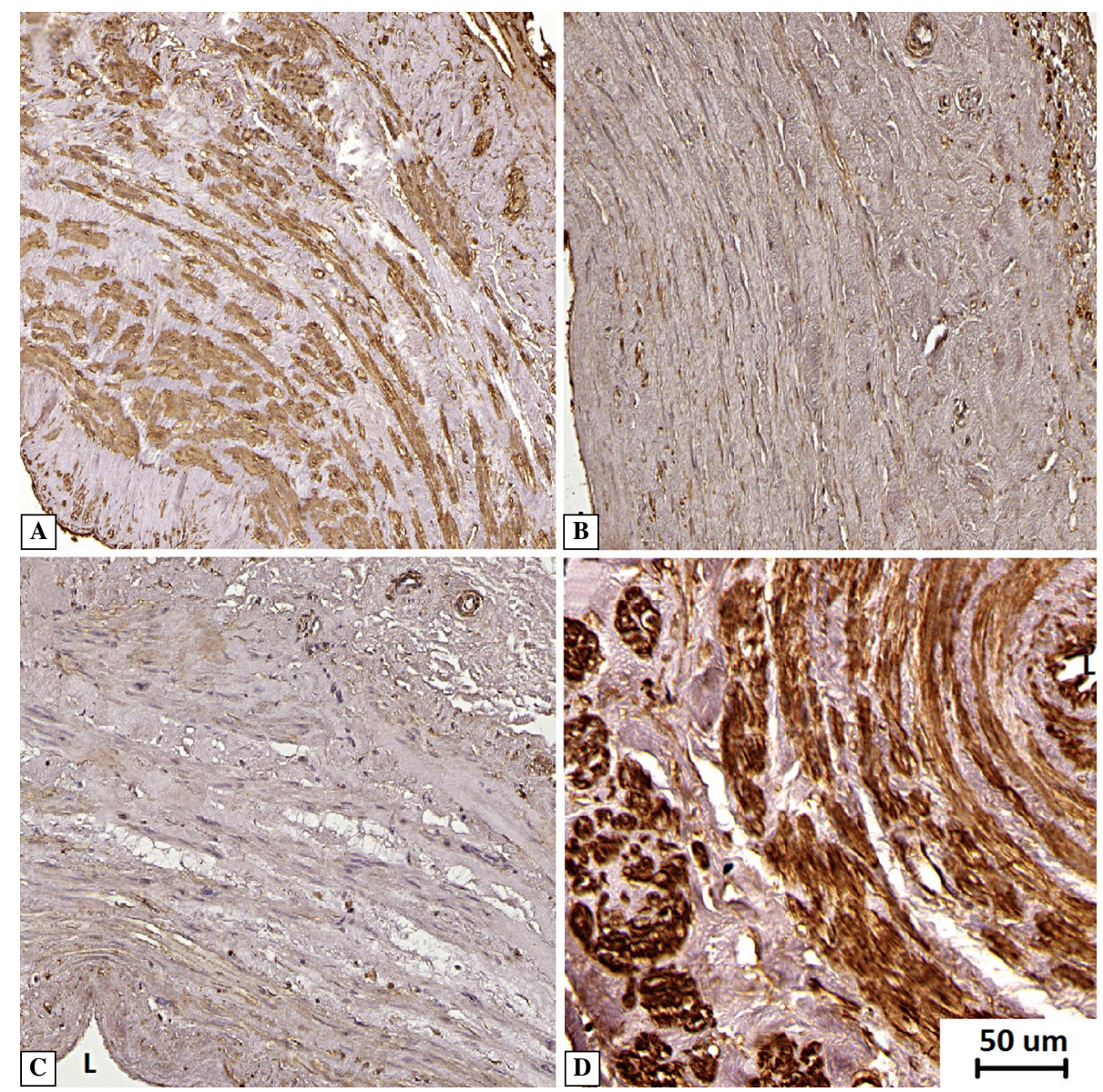

Figure 3. Immunoreactivity of MMP-2 and TIMP-2 in the tunica media of the saphenous vein grafts of middle age and elderly patients. A, C. Section of saphenous vein (SV) segment of 47-year-old patient. Strong expression of MMP-2 (IRS score 8) (A), and no expression of TIMP-2 (IRS score 1) (C); B, D. Section of SV of 76-year-old patient. Negative immunostaining for MMP-2 (IRS score 1) (B), and strong tissue expression of TIMP-2 (IRS score 12) (D). Abbreviations as for Figure $1 ; \mathrm{L}-$ lumen of the SV graft. Objective magnification: $\times 20$.

immunostaining of MMP-2, TIMP-2 and TIMP-3 within the tunica media as shown in Figure 1.

The tissue expression of MMP-2 and TIMPs was associated with the age of the patients. However, the correlations were negative for MMP-2 $(\mathrm{r}=-0.417$; $\mathrm{P}<0.05$ ) and positive forboth TIMPs(TIMP-2: $\mathrm{r}=0.413$, $\mathrm{P}<0.05$; TIMP-3: $\mathrm{r}=0.406, \mathrm{P}<0.05$ ).

Moderate positive correlations were found between both TIMPs and SM-MHC expression (TIMP-2 vs. SM-MHC $\mathrm{r}=0.517 ; \mathrm{P}<0.05$ and TIMP-3 vs. SM-MHC $\mathrm{r}=0.404 ; \mathrm{P}<0.05$, respectively) as well as between MMP-2 and CK-8 immunoreactivity ( $\mathrm{r}=$ $=0.517 ; \mathrm{P}<0.05)$.

\section{TEM study}

Transmission electron microscopy revealed that in the younger patients (below 50 years), SMCs were equipped with well-developed organelles of protein synthesis apparatus such as rough endoplasmic reticulum (RER) and Golgi complexes located close to the nucleus (Figure 4). Moreover, in this group of CABG patients, the cytoplasm was also filled with numerous glycogen deposits. In the ECM, wellpacked and ordered collagen fibers were observed. In the elderly population, majority of the SMCs had centrally located nuclei and markedly reduced number of intracellular structures involved in protein synthesis as well as the presence of electron dense, lamellar bodies, seen in degenerated SMCs (Figure 5). Moreover, abundant bundles of collagen fibers were seen in the ECM which disrupted a concise arrangement of SMCs, typical for the tunica media.

\section{Discussion}

We found that ageing of CABG patients has significant impact on preexisting expression in venous smooth muscle cells of a number of proteins either homeostatic or cytoskeletal. The constellation of these changes may promote better long-term SV graft pa- 


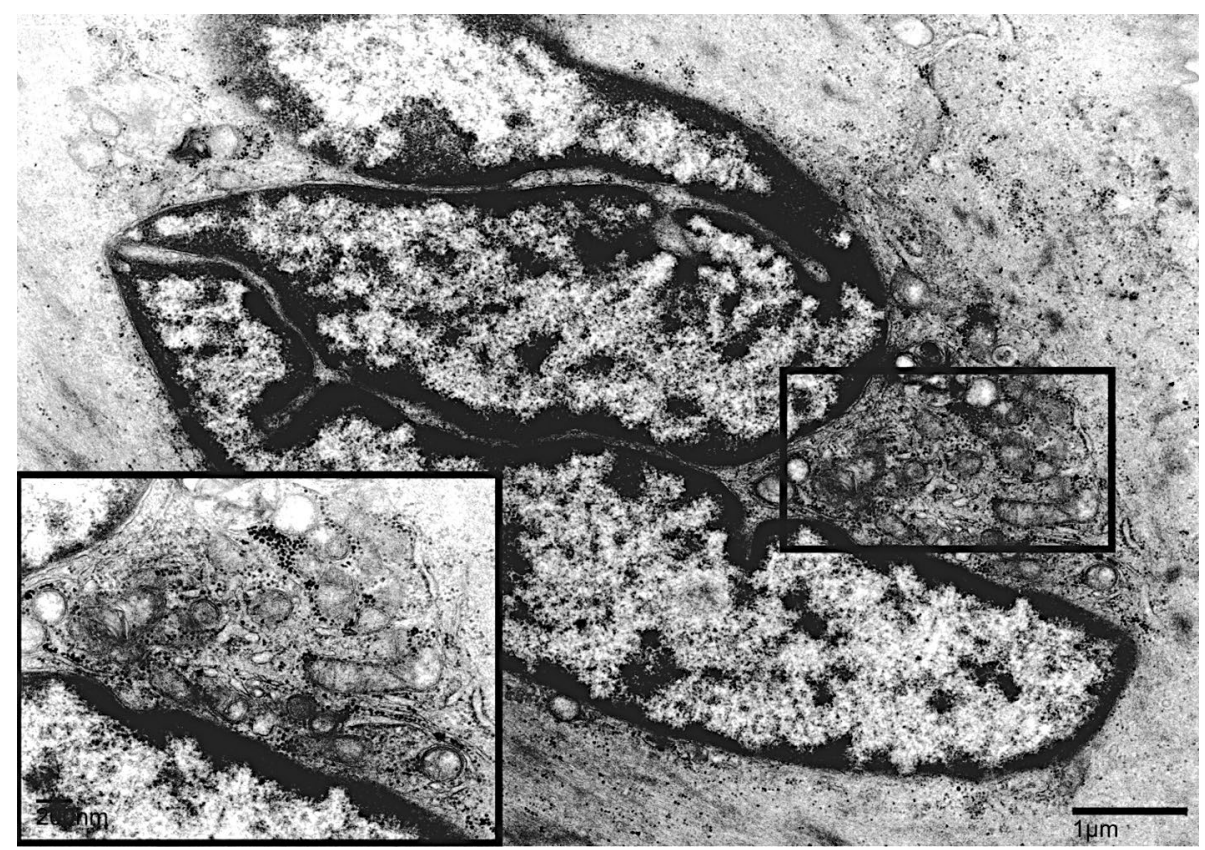

Figure 4. Transmission electron microscopic scan of tunica media from saphenous vein of 45-year-old coronary artery bypass grafting patient. Cytoplasm and nucleus of a smooth muscle cell from saphenous vein media is visible. Characteristic peripheral localization of heterochromatin in the nucleus and accumulation of cellular organelles in the area of nuclear invagination. Insert: glycogen deposits, mitochondria cristae, and few cisternae of rough and of smooth endoplasmic reticulum.

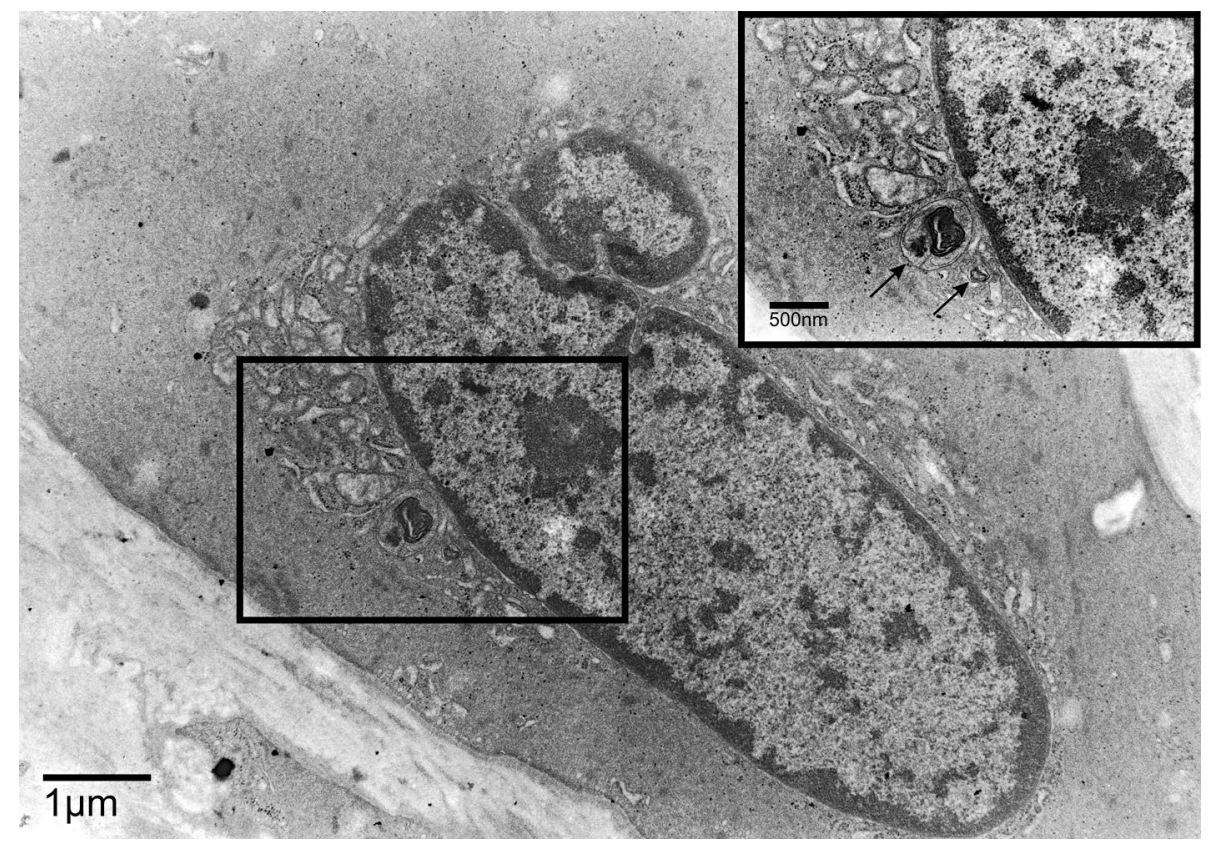

Figure 5. Transmission electron microscopic scan of tunica media from saphenous vein of 74-year-old coronary artery bypass grafting patient. Longitudinal section of smooth muscle cell from saphenous veins media shows centrally localized cell nucleus surrounded by abundant cytoplasm. Close to the nucleus accumulation of mitochondria and small portion of rough endoplasmic reticulum are visible. Insert: presence of lamellar bodies.

tency and further favorably influence outcomes after CABG with venous aortocoronary grafts. Of note, the aforementioned variations were found among the SV segments that based on their gross morphology were recognized intraoperatively as normal by experienced cardiac surgeons. 
We showed for the first time that ageing of venous SMCs is associated with altered expression of structural proteins. First of all, $\alpha$-actin immunostaining was employed to distinguish SMCs from the other cell types in the tunica media of the SV segments [12]. $\alpha$-SM actin was previously detected in all stages of SMCs development [8]. We found that ageing of CABG patients had weak impact of $\alpha$-SM actin immunostaining in the studied SMCs. However, tendency toward weaker expression with the age of $\alpha$-SM actin was noted. The expression of SM-MHC and CALP, typical proteins of well-differentiated and mature SMCs was found proportional to the age of CABG patients. Interestingly, SMCs obtained from varicose veins showed significantly higher rate of cell proliferation and migration and presented a $20 \%$ reduction in the expression of SM-MHC compared with the SMCs derived from normal veins [13]. Contrary to the aforementioned proteins, cytokeration- 8 , a marker of immature and fetal cells, found in our previous study as an independent risk factor for premature occlusion of SV grafts [14], in our study showed adverse correlation with age. Thus, preexisting variable tissue expression of marker cytoskeletal SMCs proteins within the tunica media of SV graft may suggest that younger SV grafts recipients may be at higher risk of premature venous graft failure.

We also showed for the first time that preexisting tissue immunoreactivity of one of the most potent MMPs, the MMP-2, as well as TIMPs, in the SV tunica media were also age-dependent. An earlier report by McNulty et al., demonstrated aging-related MMP-2 upregulation in the human aorta and no changes in the internal mammary artery [13]. The findings of our study suggesting that age-dependent systematical decrease in the MMP-2 tissue expression in SV wall was accompanied by increase in TIMPs immunostaining may be of clinical importance for CABG patients. Previously published studies showed that a loss of balance between MMPs and their tissue inhibitors had impact on the development of cardiovascular pathologies such as aneurysms, varices and other $[15,16]$. The degradation of ECM by MMPs, which provides a structural framework essential for the functional properties of vessel wall, was found to be a prerequisite for SMCs migration, a critical step in neointima formation [4]. Moreover, baseline level of MMP-2 in the SV wall was shown to have a strong linear correlation with the degree of luminal narrowing at 1 year after CABG [17]. Our finding of an association between MMP-2 expression and degree of differentiation of the venous SMCs in the tunica media supports the observations of Xiao et al. who showed a negative association between production of
MMP-2 and degree of differentiation of the medial SMCs in the SV segments derived from varicose veins [15]. These cells were found not only immature (probably dedifferentiated) but also were characterized by higher production of MMP-2 [15]. Thus, we can presume SV segments may be more appropriate grafts in the elderly population. However, in a view of fact that prevalence of varices increases with age of the humans it is highly possible that immature SMCs within the tunica media of varicose veins present the subpopulation of the dedifferentiated cells in a result of repeated trauma, chronic inflammation and/or higher orthostatic pressure. Having in mind this fact we tried to avoid harvesting SV segments from patients with any history of venous disorders and macroscopic abnormalities.

Moreover, our study demonstrated also increased tissue activity of TIMP-2 and TIMP-3 in the tunica media of the SV segments. Contrary to our findings in saphenous veins, no correlation between age of patient and TIMP-2 activity in the human aortic specimen had been demonstrated [13]. It was suggested that TIMP-3 was a senescence-related protein and its level was increased in Bruch's membrane in age-related macular degeneration [18]. Our finding of moderate positive correlation between tissue expression of TIMP-3 in the media of the SV grafts and the age of CABG patients is in line with the observation that TIMP-3 immunoreactivity was absent in lung and kidney specimens for all subjects below 20 years of age whereas nearly all individuals above 50 years of age exhibited strong TIMP-3 staining [19]. In our study, TIMP-3 immunostaining was present in all SV segments, but only individuals older than 40 years were enrolled.

Additionally, we have also found the signs of microstructural degeneration within the tunica media in the elderly. Our TEM study showed that in them SMCs within the tunica media lost their concise arrangement and possessed intracellular structures typical for abnormal lipid accumulation [20]. Such structures were found previously in SMCs in the $d u c$ tus arteriosus just before its physiological closure [21]. As mentioned before, neointima formation is a result of many active processes and any form of preexisting degeneration or impaired ability of SMCs to react to different local and systemic stimuli may be considered as protective [22]. This finding supports, at least partially, a marked decrease in rate of post-discharge SV graft failure in the elderly, defined as 70 years or older [14, 23].

Our results have to be considered within the context of the study's limitations. First, our study group was moderate in numbers involving 216 patients. 
We thus cannot rule out that enrollment of more individuals would increase significance and degree of correlation between estimated variables. However, it would be unlikely to alter the direction and thus overall meaning of our results. Second, we enrolled consecutive $\mathrm{CABG}$ patients, which resulted in the overrepresentation of individuals aged between 60 and 70 years of age. This might have had some impact on our findings and their generalizability to younger and older patients. Additionally, the immunohistochemical analysis of protein expression is semi-quantitative, and performed upon visual inspection with light microscopy. To minimize possible bias, two independent histologists with high expertise in immunohistochemistry analyzed the samples. Eventually, the significance of our study must be supported by long-term clinical and angiographic follow up. The preliminary results suggest that preexisting strong expression of TIMPs accompanied by weak expression of MMP-2 in the tunica media of the SV segments are associated with favorable late CABG outcomes [24]. However, longer follow-up of larger cohorts of patients is needed to clinically support our findings.

Concluding, our study demonstrated that ultrastructural and functional signs of degeneration of SMCs within the tunica media of the SV segments used as aortocoronary bypass grafts are associated with ageing of CABG patients. A progressive preexisting decrease in the expression of MMP-2 and CK-8 accompanied by increased immunostaining of TIMPs, CALP and SM-MHC with the age of patients may predict higher patency rate of venous aortocoronary bypass grafts and clinically better outcomes in the elderly population.

\section{References}

1. Vaupel JW. Biodemography of human ageing. Nature. 2010;464:536-542. doi: 10.1038/nature08984.

2. Haskett D, Johnson G, Zhou A, Utzinger U, Vande Geest J. Microstructural and biomechanical alterations of the human aorta as a function of age and location. Biomech Model Mechanobiol. 2010;9:725-736. doi: 10.1007/s10237-010-0209-7.

3. Saunderson CED, Brogan RA, Simms AD, Sutton G, Batin PD, Gale CP. Acute coronary syndrome management in older adults: guidelines, temporal changes and challenges. Age Ageing. 2014;43:450-455. doi: 10.1093/ageing/afu034.

4. HassantashSA,BikdeliB,KalantarianS,SadeghianM,HalehA Pathophysiology of aortocoronary saphenous vein bypass graft disease. Asian Cardiovasc Thorac Ann. 2008;16:331-336. doi: 10.1177/021849230801600418.

5. Muto A, Model L, Ziegler K, Eghbalieh SD, Dardik A. Mechanisms of vein graft adaptation to the arterial circulation - Insights into the neointimal algorithm and management strategies. Circ J. 2010;74:1501-1512. doi: 10.1253/circj.CJ$10-0495$.

6. Johnson JL, van Eys GJ, Angelini GD, George SJ. Injury induces dedifferentiation of smooth muscle cells and increased matrix-degrading metalloproteinase activity in human saphenous vein. Arterioscler Thromb Vasc Biol. 2001;21:1146-1151. doi: 10.1161/hq0701.092106.
7. Raffetto JD, Khalil RA. Matrix metalloproteinases and their inhibitors in vascular remodeling and vascular disease. Biochem Pharmacol. 2008;75:346-359. doi: 10.1016/j. bcp.2007.07.004.

8. Wang Z, Rao PJ, Castresana MR, Newman WH. TNF- $\alpha$ induces proliferation or apoptosis in human saphenous vein smooth muscle cells depending on phenotype. Am J Physiol Heart Circ Physiol. 2005;288:H293-H301. doi: 10.1152/ aipheart.00165.2004.

9. Harris LJ, Abdollahi H, Zhang P, McIlhenny S, Tulenko TN, DiMuzio PJ. Differentiation of adult stem cells into smooth muscle for vascular tissue engineering. J Surg Res. 2011;168:306-314. doi: 10.1016/j.jss.2009.08.001.

10. Remmele W, Stenger HE. Recommendation for uniform definition of an immunoreactive score (IRS) for immunohistochemical estrogen receptor detection in breast cancer tissue. Pathologie. 1987;8:138-140. PMID: 3303008.

11. Chung MK. Correlation Coefficient. In: Salkin NJ, ed. Encyclopedia of Measurement and Statistics. London: Sage Publications; 2007:189-201.

12. Zhu TX, Lan B, Meng LY et al. ECM-related gene expression profile in vascular smooth muscle cells from human saphenous vein and internal thoracic artery. J Cardiothoracic Surg. 2013;8:155. doi: 10.1186/1749-8090-8-155.

13. McNulty M, Spiers P, McGovern E, Feely J. Aging is associated with increased matrix metalloproteinase-2 activity in the human aorta. Am J Hypertens. 2005;18:504-509. doi: 10.1016/j. amjhyper.2004.11.011.

14. Perek B, Malinska A, Ostalska-Nowicka D et al. Cytokeratin 8 in venous grafts - a factor of unfavorable long-term prognosis in CABG patients. Cardiol J. 2013;20:583-591. doi: 10.5603/ CJ.2013.0142.

15. Xiao Y, Huang Z, Yin H, Lin Y, Wang S. In vitro differences between smooth muscle cells derived from varicose veins and normal veins. J Vasc Surg. 2009;50:1149-1154. doi: 10.1016/j. jvs.2009.06.048.

16. Morris DR, Biros E, Cronin O, Kuivaniemi H, Golledge J. The association of genetic variants of matrix metalloproteinases with abdominal aortic aneurysm: a systematic review and meta-analysis. Heart. 2014;100:295-302. doi: 10.1136/ heartjnl-2013-304129.

17. Kon ZN, White C, Kwon MH et al. The role of preexisting pathology in the development of neointimal hyperplasia in coronary artery bypass grafts. J Surg Res. 2007;142:351-356. doi:10.1016/j.jss.2007.03.062.

18. Kamei M, Hollyfield JG. TIMP-3 in Bruch's membrane: changes during aging and in age-related macular degeneration. Invest Ophthalmol Vis Sci. 1999;40:2367-2375. PMID: 10476804.

19. Macgregor AM, Eberhart CG, Fraig M, Lu J, Halushka MK. Tissue inhibitor of matrix metalloproteinase-3 levels in the extracellular matrix of lung, kidney, and eye increase with age. J Histochem Cytochem. 2009;57:207-213. doi: 10.1369/ jhc. 2008.952531.

20. Lajoie P, Guay G, Dennis JW, Nabi IR. The lipid composition of autophagic vacuoles regulates expression of multilamellar bodies.J Cell Sci. 2005;118:1991-2003. doi: 10.1242/jcs.02324.

21. Toda T, Tsuda N, Takagi T, Nishimori I, Leszczynski D, Kummerow F. Ultrastructure of developing human ductus arteriosus. J Anat. 1980;131:25-37. PMID: 7440403.

22. Mieno S, Boodhwani M, Clements RT et al. Aging is associated with an impaired coronary microvascular response to vascular endothelial growth factor in patients. $J$ Thorac Cardiovasc Surg. 2006;132:1348-1355. doi: 10.1016/j.jtcvs. 2006.08.043.

23. Perek B, Malinska A, Stefaniak S et al. Predictive factors of late venous aortocoronary graft failure: ultrastructural studies. PLoS One. 2013;8:e70628. doi: 10.1371/journal.pone.0070628.

24. Perek B, Malinska A, Misterski M et al. Preexisting high expression of matrix metalloproteinase-2 in tunica media of saphenous vein conduits is associated with unfavorable long-term outcomes after coronary artery bypass grafting. Biomed Res Int. 2013;2013:730721. doi: 10.1155/2013/730721.

Submitted: 13 January, 2016 Accepted after reviews: 30 June, 2016 Available as AoP: 12 July, 2016 